\title{
From Darcy to Gaussian to fully mobilised grain flow in a confined channel
}

\author{
Miles Morgan ${ }^{1, *}$ and Bjфrnar Sandnes ${ }^{1, * *}$ \\ ${ }^{1}$ College of Engineering, Swansea University, Bay Campus, Fabian Way, Swansea, SA1 8EN, UK
}

\begin{abstract}
Fluid-driven grain flow through a confined channel filled with non-buoyant grains is herein observed to exist in three regimes according to total imposed flow rate. (1) At low imposed flow rates, no grain flow occurs as the fluid stress is insufficient to mobilise the grains and Darcy flow is observed. (2) At a sufficient imposed flow rate, grains begin to flow at the top of the channel with self-similar Gaussian velocity profiles that become faster and encroach deeper into the channel with increased flow rate. (3) At high flow rates, significant grain flow occurs at the base of the channel, distorting the Gaussian profile, resulting in a gradual transition towards a more symmetric, full-channel flow. Each regime, and the transitions between them, is discussed in relation to experimental grain velocity measurements.
\end{abstract}

\section{Introduction}

Confined grain flows are prevalent in a range of industrial and natural settings including flow in fractures [1], sinkhole formation [2] and silos [3]. In the case of silos and hoppers, Beverloo's Law is considered to accurately describe the flow rate of grains through an aperture, however it is still the subject of new research $[4,5]$ and breaks down somewhat in submerged silos [6]. Dry silos with a lateral orifice can also adhere to Beverloo's Law, although flow ceases when the silo walls become too thick [7, 8]. If driven by fluid however, flow of non-buoyant grains can occur through a lateral orifice and into a horizontal conduit [9].

The most widely studied fluid-driven grain flows are perhaps bedload and Poiseuille flow. Bedload transport involves the shearing of a granular bed by fluid above. The onset of grain flow is often linked to the Shields parameter, a ratio of fluid and gravitational stress, while the resulting grain velocity profiles are said to decay exponentially with bed depth [10, 11]. Conversely, Poiseuille flow of grains typically concerns neutrally buoyant granular material with velocity profiles comparable to those of Bingham-style plug flows [12, 13]. However, we recently reported that grains filling a confined horizontal channel, when driven by fluid appear to exhibit self-similar Gaussian velocity profiles that become faster and penetrate deeper into the bed as the flow rate is increased [9]. This contrast to bedload flow was linked to the contact between the granular material and the upper confining wall, not unlike Couette grain flows which also betray decaying Gaussian velocities centred at the boundary. Not before considered however, is how the flow develops beyond the Gaussian regime in a confined geometry, as the flow rate is

*e-mail:m.1.morgan@swansea.ac.uk

**e-mail: b.sandnes@swansea.ac.uk

A video is available at https://doi.org/10.48448/220d-t322 increased further and the channel is fully mobilised, precluding Gaussian profiles.

This experimental work utilises velocity profile measurements to examine the flow evolution of non-buoyant grains filling a confined channel. Three regimes are considered in terms of total imposed flow rate: (1) at low flow rates where the fluid stress is insufficient to mobilise any grains (2) moderate flow rates where grains in only part of the channel are mobilised and (3) high flow rates where grains throughout the whole channel are in motion.

\section{Methodology}

Grain flow was carried out in a narrow cell consisting of two chambers connected by a $55 \mathrm{~mm}$ long horizontal channel with a rectangular cross section of width $b=1.00 \pm 0.03 \mathrm{~mm}$ and various heights $h=2,3,4$ and $6 \mathrm{~mm}$, pictured in Figure 1. The experiment was initialised by filling the entire cell with water before injecting a suspension of water and soda lime glass beads (sieved to between 100 and $200 \mu \mathrm{m}$ ) into the first (supply) chamber which, when settled, filled both the chamber and the horizontal channel. Water was then injected with a Harvard PHD Ultra syringe pump through an inlet at the top of the supply chamber which, if sufficient, mobilised the grains filling the channel, carrying them into the second (outlet) chamber where they were deposited. Excess water was allowed to exit through an outlet at the top of the outlet chamber. Grain flow in the channel was captured equidistant between the two chambers with a Nikon $1 \mathrm{~J} 4$ camera with a $60 \mathrm{~mm}$ macro lens at between $120 \mathrm{fps}$ and 1200 fps. Particle image velocimetry was then performed with PIVlab software in Matlab [14]. Measurements were averaged over $2 \mathrm{~s}$ of time and at least $2 \mathrm{~mm}$ of the channel length. The grain depletion in the supply chamber was captured simultaneously with a second camera (Nikon 1 J2) and used to measure volumetric grain flow rate $Q_{g}$. 


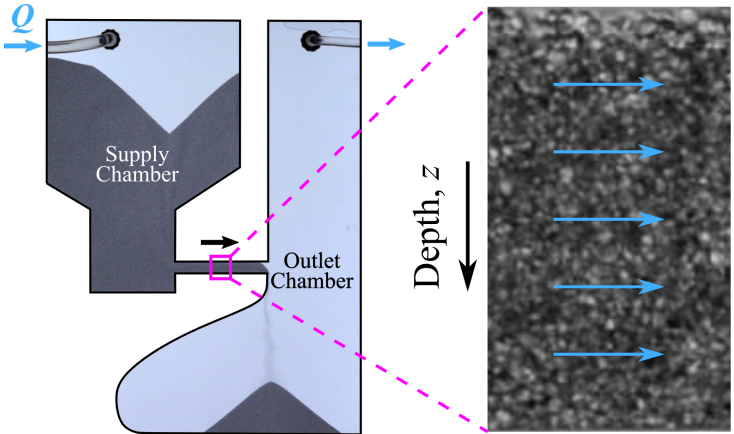

Darcy

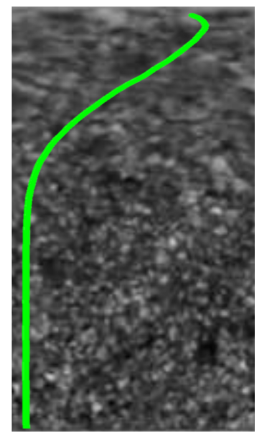

Gaussian

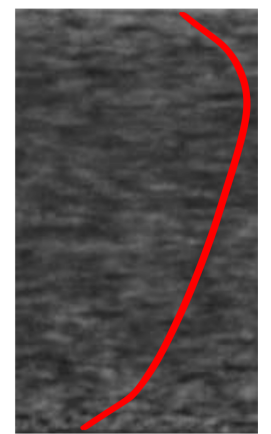

Full Flow

Figure 1. The flow cell (left) and images of grain flow in the channel for each of the three flow regimes (right). The blue arrows represent the flow of interstitial fluid through the pore space of the static packing and the green and red lines show the grain velocity profiles of the Gaussian $(0.5 \mathrm{ml} / \mathrm{min})$ and full flow $(15 \mathrm{ml} / \mathrm{min})$ regimes respectively in a $3 \mathrm{~mm}$ channel.

Filling with a known volume of granular material confirmed a static grain filling fraction of $\phi=0.579 \pm 0.001$.

\section{Results and Discussion}

Flow through the channel was observed to occur in three regimes with varying flow rate $Q$. At the lowest imposed flow rates there was no grain flow and the injected liquid flowed through the pore space of the static grain packing. Above a critical flow threshold $Q_{0}$, grain flow occurred just at the top of the channel, increasing in velocity and penetrating deeper into the channel with increased $Q$, whilst maintaining self-similarity. As the flow rate was increased beyond a second threshold $Q_{1}$ the velocity at the base of the channel became significant and grains were seen to flow trough the whole depth of the channel, with velocity profiles no longer being self-similar for different flow rates. These behaviours may be quantified by considering the effective flowing depth of the channel $z_{f}$, defined here as the depth at which the grain velocity falls more two orders of magnitude lower than its maximum, $z_{f}=z\left(\frac{v_{\max }}{100}\right)$.

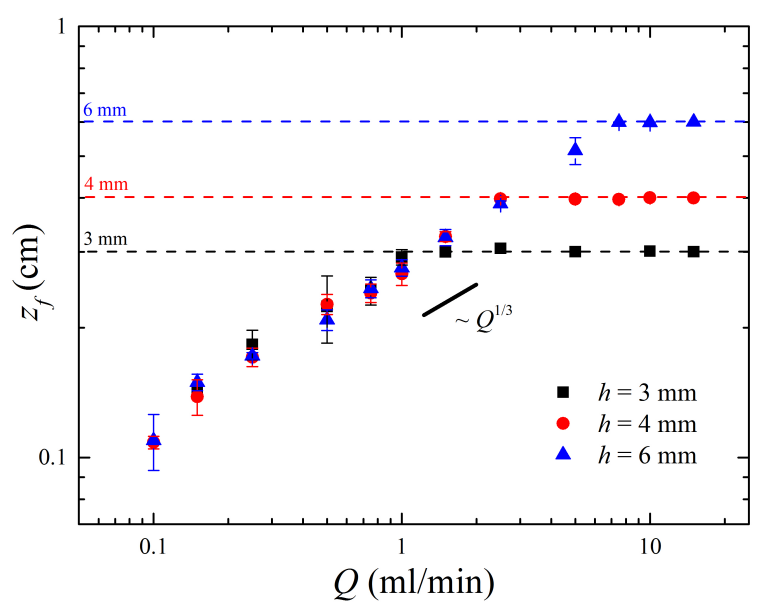

Figure 2. The depth to which grains are mobilised as a function of flow rate $Q$ for various channel heights $h$. The dashed lines represent the full channel height $h$ for each data set.
This is plotted in Figure 2 as a function of flow rate for various channel heights. For all $h$ displayed, $z_{f}$ increases with $Q$ in a manner consistent with a power law of exponent approximately $\sim 1 / 3$. This demonstrates how the flow gradually penetrates the packing of the channel and is consistent with previous findings [9]. Once $z_{f}=h$ and $Q \geq Q_{1}$ all parts of the channel satisfy the condition $v(z)>v_{\text {max }} / 100$, hence there is no more packing to mobilise and full channel flow occurs.

Above the initial threshold $Q_{0}$, the grain flow rate $Q_{g}$ measured by monitoring the depletion of grains in the supply chamber - was observed generally to increase linearly with $Q$, both before and after full channel mobilisation at $Q_{1}$ and despite the considerable changes in flow shape. Each flow regime is discussed in the following sections, predominantly considering a channel height of $h=3 \mathrm{~mm}$.

\subsection{Darcy Regime}

At low imposed flow rates no grain flow was observed and the injected liquid flowed through the granular packing in a manner described by Darcy's Law. In such cases the stress from the injected fluid was not sufficient to mobilise the non-buoyant, frictional grains. The fluid pressure gradient through the packing according to Darcy's Law is

$$
|\nabla P|=\frac{\eta Q}{k A}
$$

where $Q$ is imposed total flow rate, $\eta$ is interstitial fluid viscosity, $k$ is permeability and $A$ is the cross sectional area of the channel [15]. If we consider the grain mobilisation flow threshold for fluid-invaded porous systems $[16,17]$ we may predict the limit of this Darcy regime. If $Q_{m}$ is the flow rate at which the fluid pressure gradient of Equation 1 overcomes the forces of gravitational friction from the grains, $|\nabla P|>\mu \rho g$, where $\mu$ is the friction coefficient, $\rho$ is buoyancy-corrected grain density and $g$ is gravitational acceleration, it follows that

$$
Q_{m}=\frac{\mu \rho g k A}{\eta}
$$




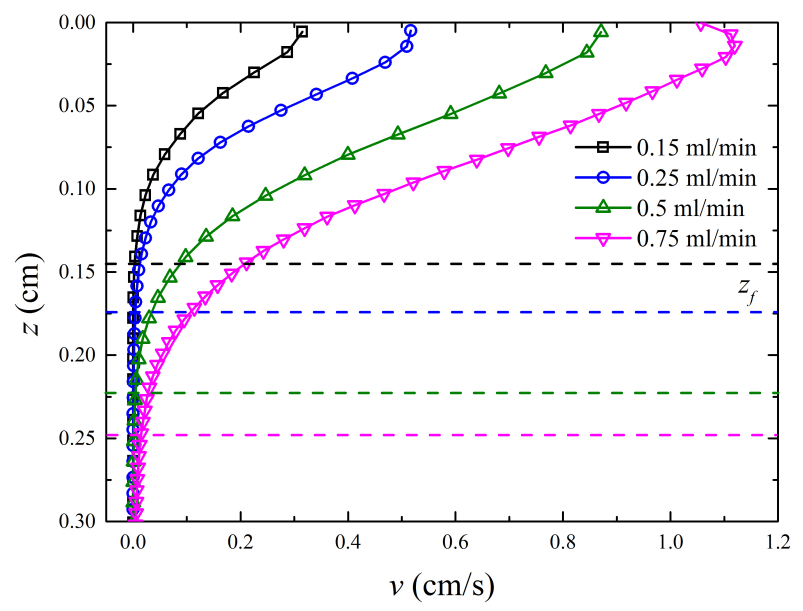

Figure 3. Velocity as a function of depth in the Gaussian flow regime for $h=3 \mathrm{~mm}$. Dashed lines correspond to $z_{f}$ shown in Figure 2.

Assuming $k=\frac{d^{2}}{180} \frac{(1-\phi)^{3}}{\phi^{2}}$ according to the Kozeny-Carman relation where $d$ is volume averaged grain diameter, measured as $133 \mu \mathrm{m}$ and the friction coefficient is taken from the angle of repose in the cell such that $\mu=\tan \left(32^{\circ}\right)$ then $Q_{m}=0.036 \mathrm{ml} / \mathrm{min}$. A direct measurement of this transition is difficult to obtain as the flow is often intermittent near the threshold, however an extrapolation of the linear grain flow rate to zero flow yields an experimental grain flow threshold of $Q_{0}=0.05 \mathrm{ml} / \mathrm{min}$, comparable to that predicted by Equation 2, corresponding to a pore velocity of $0.066 \mathrm{~cm} / \mathrm{s}$.

\subsection{Gaussian Regime}

Once the flow threshold $Q_{0}$ is surpassed, grains began to flow at the top of the channel where the gravitational stress is lowest. Figure 3 shows typical velocity profiles as a function of channel depth $z$ for a range of flow rates. As recently reported [9], the grains exhibit velocities consistent with Gaussian profiles centred at the upper wall which can be described by

$$
v(z)=v_{0} \kappa \exp \left(-\frac{1}{2}\left(\frac{z}{\sigma}\right)^{2}\right)
$$

where $v_{0}$ is the Gaussian peak, $\sigma$ is its standard deviation and $\kappa$ is an expression to account for the velocity reduction due to friction at the upper boundary. Employing a scaling of the same form to that in ref. [9], the inset of Figure 3 shows velocity profiles normalised with respect to $v_{0}$ and $\sigma$ such that $v_{0}=\alpha\left(\frac{Q}{Q_{0}}-1\right)^{0.64}$ and $\sigma=\beta\left(\frac{Q}{Q_{0}}-1\right)^{0.33}$ where $\alpha=0.22 \mathrm{~cm} / \mathrm{s}$ and $\beta=0.033 \mathrm{~cm}$. These constants are similar to those found with a $6 \mathrm{~mm}$ channel [9], with slight discrepancy possibly due to differing grain samples. Furthermore following [9], the term $\kappa=1-0.13 e^{\frac{z}{0.2 \sigma}}$ is applied here although the data at the top of the channel is limited by the resolution of the PIV measurements. Equation 3 is plotted on the collapsed velocity curve in Figure 4, normalised with respect to $v_{0}$ and $\sigma$, which demonstrates the self-similarity of this regime.

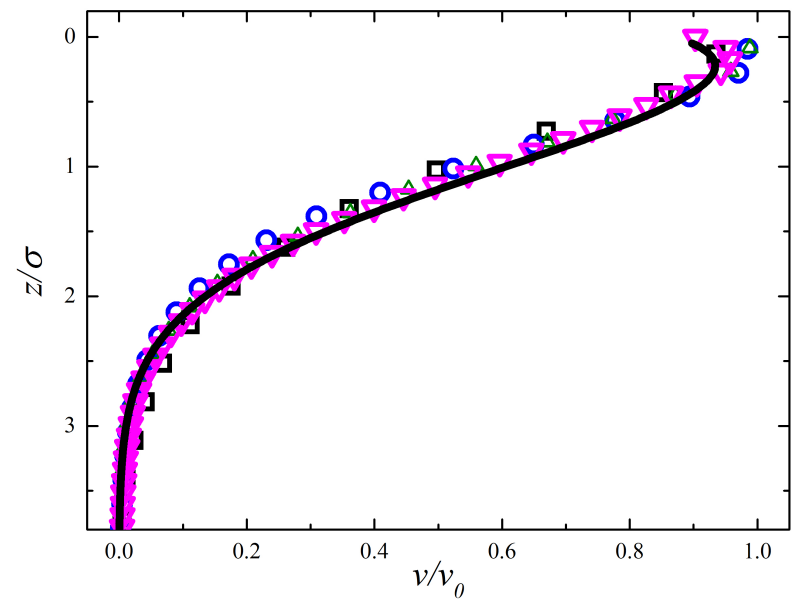

Figure 4. Velocity profiles normalised with Gaussian parameters $v_{0}$ and $\sigma$. The black solid line is Equation 3. Points are as shown by the legend in Figure 3.

As described by $v_{0}$ and $\sigma$, both the magnitude and depth to which the velocity profiles penetrate the channel increase with flow rate although they do not increase proportionally. Rather $v_{0} \sim \sigma^{2}$, suggesting that the velocity magnitude is preferentially increased with a greater imposed flow rate.

\subsection{Full Flow and Beyond}

Once the depth of the flowing region is such that it spans the entire channel and the grains at the base exhibit significant motion, the self-similarity of the Gaussian regime ceases and the velocity profiles begin to distort. Figure 5 shows velocities during this regime, normalised with maximum velocity $v_{\max }$ and channel height $h$. The concavity of the Gaussian tail at $0.75 \mathrm{ml} / \mathrm{min}$ is gradually reduced until there is no longer a point of inflection, seen for $h=3 \mathrm{~mm}$ at 5 and $10 \mathrm{ml} / \mathrm{min}$, where near-constant shear rates are

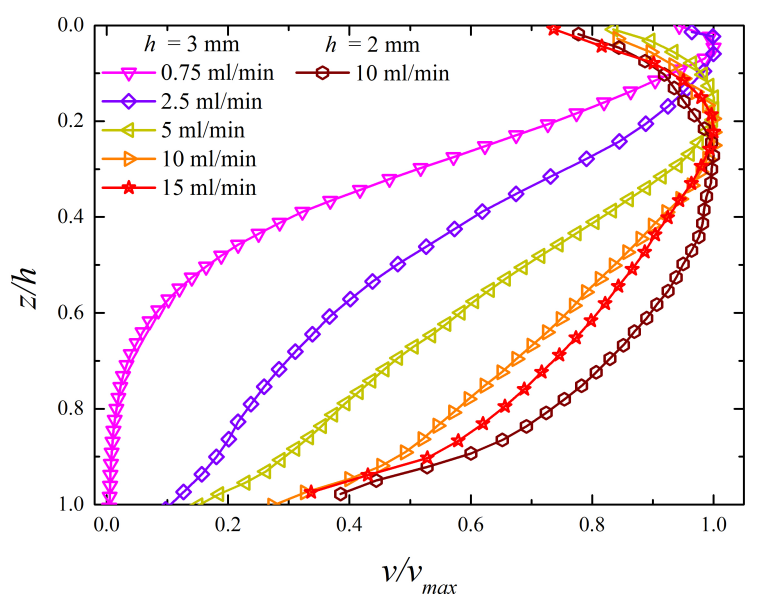

Figure 5. Evolution of velocity profiles normalised with $v_{\max }$ and $h$ as the flow rate is increased beyond the Gaussian regime and grain flow occurs in the whole channel. 
observed for much of the channel. As the flow rate is increased further the profiles become more akin to those found in Poiseuille and plug flows, suggesting velocity symmetric about the channel centre may be achieved with a high enough flow rate - once gravitational forces become insignificant - though experimental limitations prevent the present work from exploring this.

The flow rate $Q_{1}$ at which full channel flow occurs appears to be dependent on the height of the channel $h$, as shown in Figure 2 and we may postulate this dependence. As noted in the previous section, $z_{f}$, and equivalently the length scale of the Gaussian regime $\sigma$, increase with respect flow rate such that approximately $z_{f} \sim Q^{\frac{1}{3}}$ for $Q \gg Q_{0}$. Therefore at the transition to full flow $Q=Q_{1}$ where $z_{f}=h$ it follows that $Q_{1} \sim h^{3}$. This strong dependence suggests that the regime in which flow occurs at a particular flow rate may be effectively controlled with channel size.

\section{Conclusions}

Fluid-driven flow in confined channels was seen to occur in three regimes with varying flow rate and channel height. At low imposed flow rates there was no grain flow and the injected liquid flowed through the static packing as described by Darcy's law. Once a critical imposed flow rate was exceeded and the force of friction acting on the grains was overcome, grain flow occurred at the top of the channel in Gaussian velocity profiles. As the flow rate gradually increased these self-similar profiles became faster and penetrated deeper into the packing, mobilising a greater proportion of the channel. The third flow regime was established when the flow rate was high enough to mobilise grains in the entire channel; here the Gaussian shape became distorted and was no longer self-similar with respect to flow rate, instead appearing to approach a more symmetric shape, possibly towards a plug flow, as gravitational forces became less influential.

While the point of transition between these regimes was discussed, a comprehensive theory describing them is lacking and future work may consider this. Additionally, imposing higher flow rates to observe the continued evolution of the flow profiles to purely symmetric flow would be noteworthy, with pressure and filling fraction measurements to enhance understanding. The experimental configuration described here may also be employed for future studies of dense granular suspensions, like those found in such natural phenomena as erosion.

\section{Acknowledgements}

We thank James Campbell, Deren Ozturk, Dawang Zhang and Martin Monloubou for their discussions. This work was supported by the Engineering and Physical Sciences Research Council EPSRC grant EP/S034587/1.

\section{References}

[1] S. Guo, D.Z. Zhu, J. Hydraul. Eng. 143, 06017008 (2017)

[2] F. Gutiérrez, J. Guerrero, P. Lucha, Environ. Geol. 53, 993 (2008)

[3] R. Nedderman, U. Tüzün, S. Savage, G. Houlsby, Chem. Eng. Sci. 37, 1597 (1982)

[4] S. Dunatunga, K. Kamrin, J. Fluid Mech. 779, 483 (2015)

[5] J. Wan, F. Wang, G. Yang, S. Zhang, M. Wang, P. Lin, L. Yang, Powder Technol. 335, 147 (2018)

[6] T.J. Wilson, C.R. Pfeifer, N. Mesyngier, D.J. Durian, Papers Phys. 6, 1 (2014)

[7] A. Medina, D. Cabrera, A. López-Villa, M. Pliego, Powder Technol. 253, 270 (2014)

[8] Y. Zhou, P.Y. Lagrée, S. Popinet, P. Ruyer, P. Aussillous, J. Fluid Mech. 829, 459 (2017)

[9] M.L. Morgan, D.W. James, A.R. Barron, B. Sandnes, Phys. Fluids 32, 113309 (2020)

[10] M. Houssais, C.P. Ortiz, D.J. Durian, D.J. Jerolmack, Nat. Commun. 6, 1 (2015)

[11] B. Allen, A. Kudrolli, Phys. Rev. Fluids 2, 1 (2017)

[12] P. Jop, Y. Forterre, O. Pouliquen, Nature 441, 727 (2006)

[13] M. Tankeo, P. Richard, É. Canot, Granul. Matter 15, 881 (2013)

[14] W. Thielicke, E.J. Stamhuis, J. Open Res. Softw. 2, e30 (2014)

[15] J. Bear, Dynamics of Fluids in Porous Media (Elsevier, 1972)

[16] B. Sandnes, E. Flekkøy, H. Knudsen, K. Måløy, H. See, Nat. Commun. 2, 288 (2011)

[17] J.M. Campbell, D. Ozturk, B. Sandnes, Phys. Rev. Appl. 8, 064029 (2017) 